\title{
Application of Direct Peak Analysis to Energy Dispersive X-Ray Fluorescence Spectra
}

Kirk K.Nielson

July 1977

Prepared for the Energy Research and Development Administration under Contract EY-76-C-06-1830

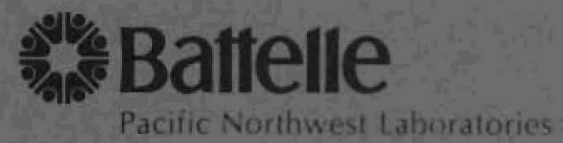




\section{NOTICE}

This report was prepared as an account of work sponsored by the United States Government. Neither the United States nor the Energy Research and Development Administration, nor any of their employees, nor any of their contractors, subcontractors, or their employees, makes any warranty, express or implied, or assumes any legal liability or responsibility for the accuracy, completeness or usefulness of any information, apparatus, product or process disclosed, or represents that its use would not infringe privately owned rights.

PACIFIC NORTHWEST LABORATORY

operated by

BATTELLE

for the

ENERGY RESEARCH AND DEVELOPMENT ADMINISTRATION

Under Contract EY-76-C-06-1830

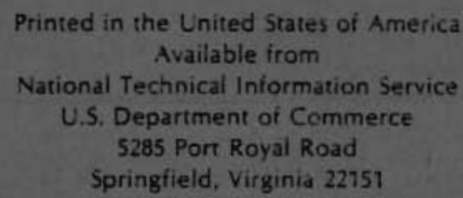

Price: Printed Copy \$__ Microfiche 53.00

$\begin{array}{cc}\text {-Pages } & \text { NTIS } \\ 001-025 & \text { Selling Price } \\ 026-050 & \$ 4.50 \\ 051-075 & 55.00 \\ 076-100 & \$ 5.50 \\ 101-125 & \$ 6.00 \\ 126-150 & \$ 6.50 \\ 151-175 & 57.00 \\ 176-200 & 57.75 \\ 201-225 & \$ 8.50 \\ 226-250 & 58.75 \\ 251-275 & 59.00 \\ 276-300 & \$ 10.00 \\ & \$ 10.25\end{array}$




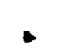


APPLICATION OF DIRECT PEAK ANALYSIS TO

ENERGY DISPERSIVE X-RAY FLUORESCENCE SPECTRA

\author{
K. K. Nielson \\ Battelle \\ Pacific Northwest Laboratories \\ Richland, Washington 99352
}

\begin{abstract}
A modified Cove11 method for direct peak analys is has been applied to energy dispersive $X$-ray fluorescence spectra. The method is background independent and is well-suited to computerized data reduction. It provides acceptable precision, minimizes errors from instrumental gain shift, and permits peak overlap correction. Peak overlap errors exhibit both positive and negative nodes as a function of peak separation distance, and are corrected using concentration ratios determined from thin, single-element standards. Peak precisions and overlaps are evaluated as a function of window width to aid in width selection. Least-square polynomial smoothing prior to peak analysis significantly improves peak area precisions without significantly affecting their accuracies.
\end{abstract}


.

. 
BNWL-2277

$\mathrm{UC}-37$

\author{
APPLICATION OF DIRECT PEAK ANALYSIS TO \\ ENERGY DISPERSIVE X-RAY FLUORESCENCE SPECTRA \\ K. K. Nielson \\ Battelle \\ Pacific Northwest Laboratories \\ Richland, Washington 99352
}

In order to analyze large numbers of samples economically using energy dispersive $X$-ray fluorescence $(X R F)$, some degree of automation in the data reduction system is necessary. A major problem in automating the system is frequently that of obtaining reliable net photopeak areas from the digital data without human interaction. Although numerous automatic peak analys is methods have been reported in the literature, few are ideal for XRF spectral analysis. Most photopeak analys is methods were originally developed for gamma-ray spectra and thus neglect two important differences found in XRF spectra: (a) the presence of an exciting source ${ }^{1}$ and matrix effects ${ }^{2}$ in XRF results in peak and background intensities which are not simple sums of single element spectra; and (b) the high ratio of peak-defining regions to background-defining regions in XRF spectra frequently precludes any direct measure of spectral background from minima between peaks. Although techniques such as peak stripping ${ }^{3}$ by least squares methods have been effectively utilized, their complexity and limitations may appear formidable if resources or experience are 1 imited. Recent literature contains widely varying suggestions for improved background calculations, ${ }^{4-10}$ including manual background subtraction. 11 our experience in using both laboratory and portable in situ XRF systems has indicated that a simple, direct peak analysis method avoids 
. 
the complexities and occasionally the errors of many other methods and yields results of sufficient precision for most routine applications.

This paper describes a modification of Covell's direct peak analysis method $^{12}$ and examines several parameters affecting its use in energy dispersive $X$-ray fluorescence. The modification permits reliable peak analys is and overlap corrections in the presence of gain shifts and aids in systematically choosing boundaries for peak integration windows. The effects of various integration window widths, spectral smoothing parameters and peak location methods on the precision and accuracy of the resulting net peak areas are evaluated. Use of alpha coefficients for peak overlap corrections is proposed for use with the modified Covell method.

\section{THEORY}

Net peak areas measured after background subtraction exceed those measured by the Covell method as illustrated in Figure 1. As illustrated, the Covel1 net area depends on a pseudo "background" defined by the 1 ine connecting the intensities at the two boundaries of the integration window. However, the resulting partial net peak area is a reproducible fraction of the total net peak area for a given window location and in the absence of gain shifts or changes in detector resolution. It is also independent of the linear spectral background beneath the peak. The Covell net area is defined as

$$
N=\underset{\substack{\sum_{i=n_{1}} \\ n_{2}}}{\left(\frac{1}{2}\left(n_{2}-n_{1}+1\right)\left(a_{n_{1}}+a_{n_{2}}\right),\right.}
$$




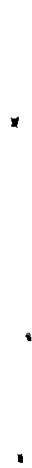


where $a_{i}$ are the counts in each channel and $n_{1}$ and $n_{2}$ are the numbers of the channels which define the integration window. Due to its simplicity, the Covell method is ideally suited for manual peak integration or for automatic integration by multichannel analyzers having this capability.

Although modern $\mathrm{Si}(\mathrm{L} i) \mathrm{X}$-ray spectrometers have dependable energy resolution at low to moderate count rates, their gain stability is less reliable. especially over long time periods or at widely varying count rates. Gain shifts affect the accuracy of Covell peak areas and affect to an even greater extent the accuracy of peak overlap corrections. Since most multielement $x$-ray fluorescence analysis relies on accurate correction of peak interferences, a modification of the usual use of Covell's method is proposed.

The proposed method depends on known $X$-ray energies and linear energy vs. channel and width vs. energy functions to define integration windows in terms of channel numbers. Intensities at the exact window boundaries are then interpolated from the intensities of the nearest spectral channels, and a Cove11-type integration is done using the exact window boundaries. This approach minimizes errors from poorly centered integration windows and permits systematic correction of peak overlap errors.

The energy (E) vs. channel ( $n$ ) function,

$$
E=s_{1} n+t_{1}
$$

is defined for each spectrum or set of spectra to provide accurate integration window placement. The constants $s_{1}$ and $t_{1}$ may often be determined individually for each sample using two known, large and well-separated peaks in the spectrum. For environmental samples, peaks from $\mathrm{Fe}\left(\mathrm{K}_{\alpha}\right)$ and from the 

coherent scattered exciting radiation are usually adequate. Alternatively, an energy calibration sample can be analyzed prior to the sample set to establish the energy scale. Peak centers for the energy calibration may be accurately determined by simple least squares methods. ${ }^{13}$

Integration window boundaries, indicated in Figure $1 b$ by $n_{1}^{\prime}$ and $n_{2}^{\prime}$, may then be computed in terms of channel number as

$$
\begin{aligned}
n_{1}^{\prime} & =n_{c}-w \\
\text { and } n_{2}^{\prime} & =n_{c}+w
\end{aligned}
$$

where $n_{c}$ is the peak location determined from its energy and Equation 2, and $W$ is the window half-width. Although any desired peak width function may be used, the simple linear function

$$
w=r\left(s_{2} E+t_{2}\right) \text {, }
$$

with $r$ equal to unity, is usually adequate. For the purposes of this study, $s_{2}$ and $t_{2}$ were defined such that $W$ approximated the standard deviation $(\sigma)$ of the $K \alpha$ peak distributions, assuming a gaussian shape. The constant $\underline{r}$ then permitted systematic study of various window widths, reported here in units of $\sigma$.

Spectral intensities at the window boundaries $n_{1}^{\prime}$ and $n_{2}^{\prime}$ are linearly interpolated using the equations

$$
\text { and } \begin{aligned}
a_{n_{1}^{\prime}} & =a_{n_{1}}+\left(n_{1}^{\prime}-n_{1}\right)\left(a_{n_{1}+1}-a_{n_{1}}\right) \\
n_{2} & a_{n_{2}}+\left(n_{2}-n_{2}^{\prime}\right)\left(a_{n_{2}-1}-a_{n_{2}}\right) \text {, }
\end{aligned}
$$

where $n_{1}$ and $n_{2}$ represent the channel numbers (integers) enclosing $n_{1}^{\prime}$ and $n_{2}^{\prime}$ as illustrated in Figure ib. The net peak area is then computed using the 

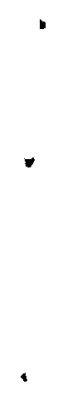

. 
modification of Equation 1,

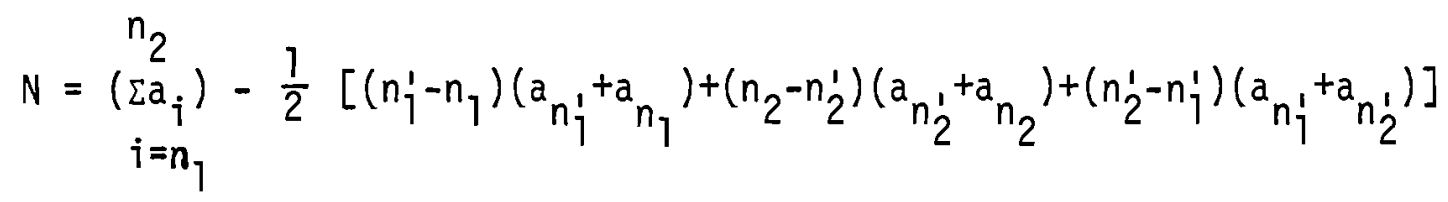

Since the intensities at the window boundaries are now defined, on the average, by 1.5 times as many channels as in Covell's method, the uncertainty of the resulting peak areas is reduced to approximately

$$
S_{N}=\sqrt{N+\frac{1}{6}\left(a_{n !}+a_{n_{2}^{\prime}}\right)\left(n_{2}^{\prime}-n_{1}^{\prime}\right)\left(n_{2}^{\prime}-n_{1}^{\prime}+1\right)}
$$

for unsmoothed spectra. If a single 5-point least-squares quadratic smoothing ${ }^{13}$ is done before peak analysis, the uncertainty is reduced to approximately

$$
S_{N}=\sqrt{N+\frac{1}{30}\left(a_{n_{1}^{\prime}}+a_{n_{2}^{\prime}}\right)\left(2 n_{2}^{\prime}-2 n_{1}^{\prime}+9\right)\left(n_{2}^{\prime}-n_{1}^{\prime}+1\right)}
$$

Equations 7 and 8 are simple modifications of Covell's original expression. ${ }^{12}$

Correction for intensity errors and non-linear background caused by interferring neighboring peaks is proposed using simple alpha-coefficient corrections,

$$
C_{i}=C_{i}+\sum \alpha_{j \neq i} C_{j}
$$

where $C_{i}$ is the directiy measured concentration, $C_{j}$ is the corrected concentration, $c_{j}$ the concentration of the interferring element, and $\alpha_{i j}$ is the overlap coefficient, defined as the apparent mass of element $i$ measured in a pure thin standard of element $j$. Similar coefficients have been previously used with other XRF peak analysis methods to correct for peak overlap. ${ }^{2}$ They may be similarly defined in terms of peak areas rather than masses if 


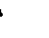

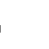

.

. 
samples are sufficiently thin that X-ray absorption is negligible. When absorption is significant, however, the corrections must be applied to the absorption-corrected peak areas or to the resulting concentrations. The $\alpha$-coefficients are often conveniently available from the standard single element analyses used in calibrating the spectrometer.

Location of peaks from spectral data has customarily been done by second-derivative spectral searches, ${ }^{14}$ cross-correlation ${ }^{15}$ or digital filtering. ${ }^{16}$ Although these methods give excellent results with well-defined peaks, our experience has illustrated the common need for a complete results matrix, i.e., determination of detection limits in all cases where the desired peaks were not significant. The present approach fulfills this need and minimizes the common errors in small peaks caused by inaccurate location of peak centers.

At least two previous modifications of Covell's method have been reported--both for $\gamma$-ray spectrometry. Sterlinski ${ }^{17}$ used the sum of several Cove11-type integrations of each peak using different widths and reported significantly improved precision in the resulting peak areas. Liebscher and Smith ${ }^{18}$ used points of inflection to define peak window boundaries for a Covell-type integration, from which an equivalent gaussian was computed. The gaussian served to exclude any non-gaussian components from the peak shape. Again, improved precision was reported. The Sterlinski and Covell methods were also included in a comparison with other peak analysis methods for gamma-ray spectral analysis. ${ }^{19}$ In a more general sense, the digital filtering of spectra as reported by $B 1$ ack ${ }^{15}$ and Robertson et a ${ }^{16}$ is closely 

related to the Cove11 method. The Covell integration acts as a digital filter which, if applied successively in single channel increments, would yield a filtered or correlation spectrum similar to those reported.

Relative precisions attainable for a hypothetical small peak as frequently encountered in XRF analysis are plotted in Figure 2 using Cove11's and Sterlinski's formulae for net peak uncertainty, and $\sqrt{N+2 B}$ and Equation 8 for the background-subtracted and modified Covell methods. A1though the curves will shift with varying peak widths, intensities, peak/ background ratios and smoothing, they give a qualitative comparison of the four methods for a typical small peak where uncertainty from counting statistics becomes large.

\section{METHODS}

In applying the modified Covel1 method to XRF spectra, it was desired to evaluate various smoothing methods, peak location methods and integration window widths. In order to compare the results of each method, a set of test data was selected which consisted of 16 spectra from replicate, sequential $1000 \mathrm{sec}$. analyses of a single $500 \mathrm{mg}, 3.18 \mathrm{~cm}$ dia. wafer of NBS orchard leaves (SRM 1571) pressed under $27000 \mathrm{~kg}$. The analyses utilized Zrk $_{\alpha, \beta}$ radiation for secondary excitation (Model 810 excitation system, Kevex, Inc., Burlingame, $\mathrm{CA}$ ) and an $80 \mathrm{~mm}^{2} \mathrm{Si}(\mathrm{Li})$ diode with $200 \mathrm{eV}$ resolution FWHM at $6.4 \mathrm{KeV}$ (Kevex, Inc.) for X-ray detection in the vacuum spectrometer. Pulse height analysis was accomplished with a computer-based analyzer system (Model 4410, Nuclear Data, Inc., Palatine, IL), and routine data 


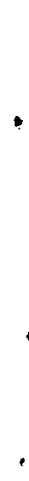


reduction was accomplished off-line with a PDP-15 computer. Spectra were collected in 1024 data channels having a nominal energy equivalent of 25 $\mathrm{eV} / \mathrm{channel}$. Thin single element standard analyses were done under identical conditions to the orchard leaf analyses and utilized $50-120 \mathrm{\mu g} / \mathrm{cm}^{2}$ deposits of single metals or binary compounds evaporated onto $0.013 \mathrm{~mm}$ mylar backings (Micromatter, Inc., Seattle, WA).

A Fortran program was written to conduct peak analyses of the test spectra in the following manner:

(a) Smoothing the raw spectral data any desired number of times up to 64 using a five-point algorithm or, optionally, using a seven-or-nine-point algorithm, ${ }^{13}$ and optionally doing the smoothing in the square-root transformed domain;

(b) Determining accurate energy/channel scale using an observed scatter peak and a known spectral peak (FeKa);

(c) Finding peak centers in terms of channel number using the energy/channel scale and known $X$-ray peak energies or, optionally, finding peak centers from spectral data using a correlation spectrum; ${ }^{16}$

(d) Finding a window width for each peak from its energy using a linear width vs. energy relationship (Equation 4);

(e) Computing window boundaries by successively adding and subtracting the appropriate width from the peak energy and converting to the nearest integral channel number $\left(n_{1}, n_{2}\right)$ or optionally, interpolating to the exact window boundaries 
. 
$\left(n_{j}, n_{2}^{-}\right)$and their corresponding intensities; and

(f) Integrating the net peak area using Covell's method as in Equation 1 or, if exact window boundaries are interpolated, using Equations 5 and 6.

The width-energy relationship in Step (d) was chosen so that most of the resulting windows were the same multiple of the FWHM of the given peak. Window widths were thus expressed in units of $\pm \sigma$, the standard deviation of the equivalent gaussian, making the FWHM equal to $1.18 \sigma$ in this notation.

\section{RESULTS AND DISCUSSION}

Since data smoothing was preparatory to peak analysis, results of various smoothing methods are considered first. Uncertainties among the 16 replicate net peak areas using the modified Covell method are plotted in Figure 3 as a function of the number of times the spectra were smoothed before peak integration. As illustrated, repeated smoothing improved the peak area precisions, and most of the improvement was achieved in the first few smoothings.

A similar plot in Figure 4 illustrates the increasing error from systematic losses in net peak areas with the repeated smoothing. Systematic error of less than a tenth of the magnitude of the random error is not shown due to its high uncertainty. Systematic error due to 5-point smoothing was always less than the corresponding random error for a given peak as long as only a few smoothings were done.

Seven and nine-point smoothings were applied to the orchard leaf spectra 


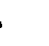

, 
with similar results to those of the five-point smoothing. For quantitative comparison, the systematic error due to one, two and four smoothings of the spectra by both seven and nine point methods are illustrated as they intersected the corresponding elemental lines from five-point smoothing in Figure 4. It is thus seen that 1, 2 and 4 seven-point smoothings cause about the same systematic error as 5,10 and 20 five-point smoothings. Similarly, 1, 2 and 4 nine-point smoothings are equivalent to 14, 29, and 58 five-point smoothings. A similar equivalence of random errors was observed after five, seven and nine-point smoothing. These results are in qualitative agreement with the noise reduction data reported by Enke and Nieman ${ }^{20}$ for multiple smoothings using various smoothing function widths. Square root transformation of the raw spectral data before smoothing was proposed by Inouye ${ }^{21}$ for proper filtering of Fourier transformed spectra. Although the smoothing methods used here ${ }^{13}$ differ considerably, the reduction in bias of the smoothing process which is achieved by first taking the square root of the raw data should be equally applicable. Table I 1ists the results of 1 and 64 five-point smoothings of the square root transformed orchard leaf spectra for comparison with direct smoothing of the raw data. Window boundaries were rounded to the nearest integer for this comparison. The peak areas are indistinguishable for a single smoothing but are noticeably different for repeated smoothing of large peaks such as $\mathrm{Fe}$ and $\mathrm{Ca}$. Use of a square root transformation in smoothing as employed here appears necessary only in cases of repeated smoothing of spectra having large, abrupt intensity changes. 


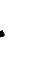

, 
The precisions of peak analyses were estimated from analyses of the 16 orchard leaf spectra using three combinations of fixed (preset) and variable (determined from spectral data) parameters as indicated in Table II. The first set of uncertainties resulted from commercially available peak analys is software in our multichannel analyzer system. Peaks were located by digital filtering, ${ }^{16}$ widths were determined from spectral peaks, and backgrounds were interpolated from minima between the peaks. The number of spectra $(n)$ in which the given peak was observed is also indicated. The second set utilized modified Covell peak integration (Equations 5 and 6) after first locating the integration windows from spectral peaks with a digital filter. ${ }^{16}$ The third set also used modified Covell peak integration, but depended on the Fek a and scatter peaks for energy scale definition and window location. Its uncertainties were general1y lower than those of the other methods. The uncertainties listed in Table I for direct Covell peak integration (Equation 1) were also generally greater than for the fixed window modified Covel1 method except after extreme smoothing. Peak areas computed from counting statistics are also listed in Table II as calculated from Equation 8. The expected uncertainties are in reasonable agreement with those observed experimentally in the preceeding column of Table II.

The accuracies of computed peak areas are more difficult to evaluate than precisions since they require reference areas for comparison. One test of accuracy is to apply the method to a variety of analyses of standard reference materials and compare the results to known concentrations. Such an evaluation has been made ${ }^{22}$ using the fixed-window modified Covell method, 


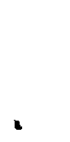


and indicated that excellent accuracy was achieved.

Further evaluation of peak accuracies relied on mean peak areas from the 16 replicate orchard leaf spectra. The areas were averaged for each of the peak analysis methods considered in Table I, and are compared in Figure 5 . The comparison is made by normalizing each mean peak area to the corresponding "total net peak intensity" determined from fundamental parameters. The total intensity was calculated as

$$
\tau\left(1-\frac{1}{J_{K}}\right) \omega_{K} \varepsilon \text { abck, }
$$

where $\tau$ is the photoelectric cross section, ${ }^{23} \mathrm{~J}_{\mathrm{K}}$ is the absorption function jump ratio, ${ }^{23} \omega_{K}$ is the fluorescent yield, ${ }^{24} \varepsilon$ is the detector efficiency, ${ }^{25}$ $\underline{a}$ is the self absorption correction factor, ${ }^{22} \underline{b}$ is the enhancement correction factor, ${ }^{22} \underline{c}$ is the concentration of the given element in the sample, and $\underline{k}$ is a normalizing factor which includes solid angles, analysis livetime and excitation intensity. It should be noted that the ratio of the $\underline{k}$ factor for the modified Covell peaks to that for the total net peaks ( $\gamma$-ray program) gives the fraction of the total net peak area used by the modified covell method (for a 1.70 window, $k_{C} / k_{T} \approx 0.7$ ).

As Figure 5 illustrates, the $\gamma$-ray program made relatively large errors in peak integration of the light elements, due primarily to poor background subtraction. The peak overlap magnitudes explain this error only in the case of As, and partially for $\mathrm{P}$ and $\mathrm{Cr}$. Systematic overlap corrections are difficult with this method due to the varying locations and widths of integration windows. Perhaps such application (or misapplication) of $\gamma$-ray analysis pro- 


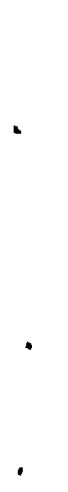


grams to crowded $X$-ray spectra has been the cause of the distrust of simple or automated spectral analysis methods by many spectroscopists. Similar difficulties in overlap corrections occur with the floating window modified Covel1 method, but are avoided when a fixed integration window is used. Most of the systematic error in Figure 5 for the modified Covell methods results from non-linear background under smal1 peaks ( $\mathrm{Cl}, \mathrm{Cr}, \mathrm{Mn}, \mathrm{Ni}, \mathrm{Cu})$, and is readily corrected in the fixed-window case by peak overlap corrections. Reasonable accuracy should also be achievable by the original Covell method (Equation 1) as long as precisely identical energy scales and windows can be assured for samples and standards.

The determination of an energy scale for each spectrum from the FeKa and scatter peaks provided a precise basis for peak location. The need for such determinations was examined by monitoring computed energy scales from routine environmental sample analysis over a 34-day period. The average energy/channel for various sets of 216 samples was constant within $0.04 \%$ (relative) when averaged over 20 sample sets. The nominal average was $s_{1}=$ $25 \mathrm{eV} /$ channel. The intercept (Equation 2) $t_{1}$ varied by over $70 \%$ relative standard deviation among the 20 sets, with a nominal average of $18 \mathrm{eV}$. In terms of actual peak centers, the standard deviation of the peak center for 18 of the sample sets was 0.4 channels. The remaining two sets had a FeKo peak shift of two channels from the mean of the others. Interference by Mn $K_{B}$ or other peaks was ruled out as an explanation when the relative concentrations of the other elements were considered. These data aid in 

evaluating the danger of peak errors due to gain shifts when using fixed channel windows over moderate time periods.

The location of peak centers from their energies and a fixed energy scale also offers the advantages of reproducible peak overlap determination and of systematic determination of detection limits in cases of insignificant peaks. Accurate determination of peak overlaps is vital in XRF analysis especially with the Covell method of peak integration which often has greater overlap magnitudes. Overlap magnitudes are qualitatively compared in Figure $6 \mathrm{a}$ and $6 \mathrm{~b}$ for conventional background subtracted integration and cove 11 integration. As illustrated, overlapping peaks cause two error regions of opposite signs in the Covell case, whose magnitudes vary with the heights and separation of the peaks involved. The net overlap error in the Covell case can be empirically shown to equal the corresponding overlap measured with identical window location and width using a thin single element standard of the interferring element. This measurement, illustrated in Figure $6 \mathrm{c}$, is conveniently accomplished by multielement analysis of the calibration spectra when thin single element standards are used.

Peak overlap errors from Covell peak integration cancel each other at a fixed peak separation distance which is apparently independent of the height of the interferring peak as illustrated in Figure 7 . These data were obtained from fixed-width integration of successive regions near the Cuk $_{B}$ peak of six analyses of a Cu standard for varying durations. The areas measured at zero displacement from the peak center were linearly related to the respective total analysis times and, as illustrated, the curve amplitudes 

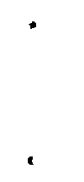
vary only with the total interferring peak intensity. Overlap corrections were thus applied as simple multiples of the interferring elemental quantity, as indicated by Equation 9.

Optimal window widths for peak integration must be chosen as a compromise between the higher precision possible with wide windows and the lower peak overlaps with narrow windows. The increased peak area measured with a wide window is illustrated in Figure 8 by the repeated integration of a single peak using various window widths and displacements. It is noted here that the peak separation distance at which the net peak overlap error is zero varies with the integration window width.

Since the magnitude of any overlap correction depends on the heights of the interferring peaks, direct evaluation of peak overlap coefficients as a function of window width is not helpful. Instead, peak overlaps expressed as relative mass error and normalized to the earth's crustal abundances 26 of the various elements are plotted versus the integration window width in Figure 9. The discontinuities in several lines occur at the zero net error points illustrated in Figures 7 and 8. Data for Figure 9 were obtained by repeated multielement analyses of single element standard spectra using varying window widths. As indicated in the figure, Co requires overlap corrections for $\mathrm{Fe}$ amounting to about 20 times its concentration and is thus difficult to accurately measure in crustal-type materials. If, however, the Co/Fe ratio were 100 times as high in a sample as in the crustal average, the correction for Fe overlap on Co would amount to only about $20 \%$ of the 

Co concentration. The peculiar shape of the $\mathrm{Ca}(K)$ curves results from the $\mathrm{K}_{\mathrm{K}_{\beta}}$ peak which is partially resolved from $\mathrm{K}_{\mathrm{K}_{\alpha}}$ and becomes included in the Ca integration window.

Since the overlap curves in Figure 9 have such widely varying slopes, no general rule of optimum window selection is possible. Statistical precision generally sets the lower window width limit at approximately $1 \sigma$, however, and widths exceeding $2.6 \sigma$ appear to unnecessarily sacrifice the accuracy of many measurements. Our experience in environmental sample analys is has indicated a window width of $1.7 \sigma$ is excellent for routine use in soil, plant, animal, water, and air particulate samples. Occasional difficulty in $\mathrm{Ni}$ measurements has been overcome by use of a $1.18 \sigma$ window. Setting window widths at different peak width multiples for different peaks was not investigated here, but may aid in optimizing peak analysis for specific sample types or conditions.

The modified Covell method described here has been used in a FORTRAN computer program for routine data reduction. ${ }^{27}$ Peak overlap corrections using Equation 9 were limited to three correction terms and were found to be generally adequate for environmental sample analysis. A related study concerning matrix corrections used the modified Covell method and gives further evidence of its precision and accuracy. 22

\section{CONCLUSIONS}

The modification of Covell's direct peak analysis method for use on XRF spectra provides a reliable, simple means of automatically obtaining net photo peak areas. Although the method does not utilize the maximum statis- 

tical precision of the peaks, its potential independence from peak location, width and background estimates more than compensates by giving reliable net peak areas. The method is particularly advantageous in cases of crowded XRF spectra and could be equally useful in similar applications such as particle induced X-ray emission.

One or two five-point smoothings of the raw data before peak analysis gave significantly better precision without significant loss in accuracy using the direct analysis method. Repeated further smoothing gave little improvement in precision, however, but lowered peak areas by about $0.1 \%$ per smoothing in the test spectra where the peak FWHM varied from 7-12 channels. Seven and nine-point smoothings were found to affect Covell peak analys is identically to selected multiple five-point smoothings. Peak overlap errors extend to a greater peak separation interval with Covell integration, but have a region of zero overlap error at a fixed peak separation interval. The overlap errors appear to be adequately corrected by simple use of $\alpha$ coefficients which are easily determined from thin single element standards. The optimum peak analys is window width is a compromise based on good precision with a wide window and minimal peak overlap with a narrow one. Environmental sample anlaysis has indicated a 1.4 FWHM (1.70) window to be routinely practical, although occasional use of 1 FWHM windows may be desirable for certain elements.

\section{ACKNOWLEDGMENTS}

The author is grateful to R. W. Sanders for first using the Covell method with X-ray fluorescence spectra and for stimulating its further study. 


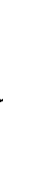


The support and suggestions of N. A. Wogman and W. D. Felix are also appreciated. This paper is based on work performed under U. S. Energy Research and Development Administration Contract EY-76-C-06-1830. 



\section{REFERENCES}

1. F. Arinc, R. P. Gardner, L. Wielopolski and A. R. Stiles, in R. W. Gould, C. S. Barrett, J. B. Newkirk and C. 0. Ruud (Eds.), Advances in X-ray Analysis, Vol. 19, Kenda11/Hunt, Dubuque, Iowa, 1976, p 367.

2. E. Lifshin in R. W. Gould, C. S. Barrett, J. B. Newkirk and C. O. Ruud (Eds.), Advances in X-ray Analysis, Vol. 19, Kenda11/Hurt, Dubuque, Iowa, $1976, \mathrm{p} 113$.

3. F. H. Schamber in T. Dzubay (Ed.), Proceedings, EPA symposium and Workshop on X-ray Fluorescence Analys is of Environmental Samples, Chapel Hill, NC, 1976.

4. P. Quittner, Nucl. Inst. Meth. 76, 115 (1969).

5. R. Grote and C. R. Cothern, X-ray Spectrom. 3, 151 (1974).

6. S. J. B. Reed, X-ray Spectrom. 4, 14 (1975).

7. S. Taczanowski, Nucl. Inst. Meth. 123, 409 (1975).

8. D. G. W. Smith, C. M. Gold and D. A. Toml inson, X-ray Spectrom. 4, 149 (1975).

9. P. J. Statham, X-ray Spectrom. 5, 16 (1976).

10. P. Van Espen and F. Adams, X-ray Spectrom. 5, 123 (1976):

11. S. Ma 1m, X-ray Spectrom. 5, 118 (1976).

12. D. F. Cove11, Ana1. Chem. 31, 1785 (1959).

13. A. Savitzky and M. J. E. Golay, Anal. Chem. 36, 1627 (1964).

14. H. P. Yule, Anal. Chem. 40, 1480 (1968).

15. W. W. Black, Nucl. Inst. Meth. 71, 317 (1969).

16. A. Robertson, W. V. Prestwich and T. J. Kennett, Nucl. Inst. Meth. 100, 317 (1972).

17. S. Sterlinski, Ana1. Chem. 40, 1995 (1968).

18. K. Liebscher and H. Smith, Anal. Chem. 40, 1999 (1968). 

19. P. A. Baedecker, Ana1. Chem. 43, 405 (1971).

20. C. G. Enke and T. A. Nieman, Anal. Chem. 48, 705A (1976).

21. T. Inouye, Nucl. Inst. Meth. 91, 581 (1971).

22. K. K. Nielson, Ana1. Chem. 49, 641 (1977).

23. W. H. McMaster, N. K. DelGrande, J. H. Mallett and J. H. Hubbell, Compilation of X-ray Cross Sections, UCRL-50174, Sec. II, Rev. I (1969).

24. W. Bambynek, B. Crasemann, R. W. Fink, H.-U. Freund, H. Mark, C. D. Swift, R. E. Price and P. V. Rao, Rev. Mod. Phys. 44, 716 (1972).

25. R. Woldseth, X-ray Energy Spectrometry, Kevex, Inc., Burl inghame, CA (1973).

26. B. Mason, "Principles of Geochemistry," 3rd Ed., John Wiley and Sons, New York, 1966, pp 45-6.

27. K. K. Nielson, SAP3: A Computer Program for X-ray Fluorescence Data Reduction for Environmental Samples, BNWL-2193, Battelle-Northwest, Richland, WA (1977). 



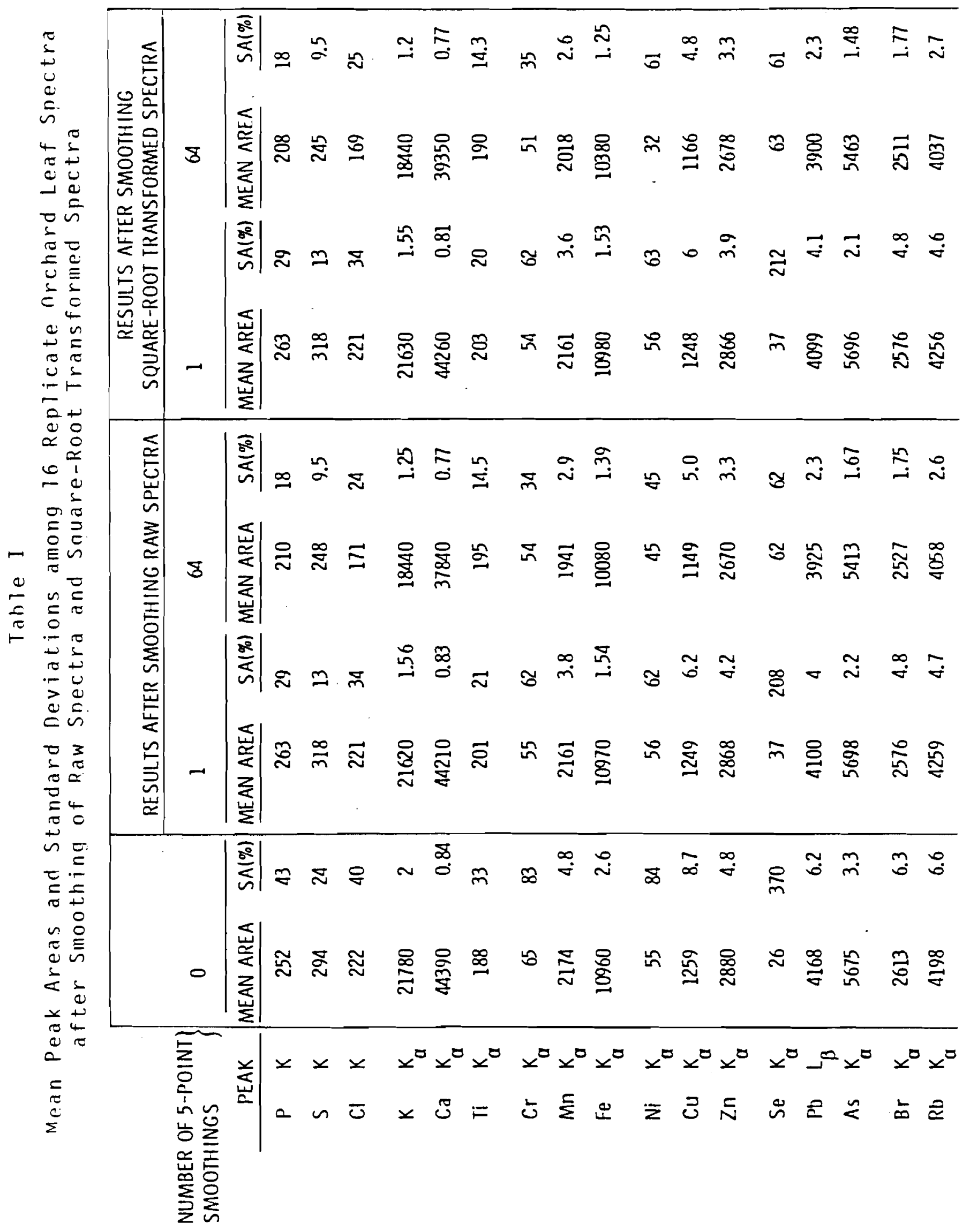


.

.

. 
Table II

Relative Peak Area llncertainties using Three Peak Analvsis Methods on 16 Renlicate Orchard Leaf Spectra

\begin{tabular}{|c|c|c|c|c|c|c|c|c|}
\hline & & $\gamma-R /$ & DGRAM & $\begin{array}{l}\text { FLOA } \\
\text { MOD }\end{array}$ & $\begin{array}{l}\text { W WINDOW } \\
\text { D COVELL }\end{array}$ & $\begin{array}{l}\mathrm{FI} \\
\mathrm{MOL}\end{array}$ & $\begin{array}{l}\text { INDOW } \\
\text { D COVELL }\end{array}$ & \\
\hline CEN & & & BLE & & IABLE & $F|\rangle$ & NERGY) & \\
\hline WII & & & $B L E$ & & $(1.7 \sigma)$ & $\mathrm{FI}$ & $(1.70)$ & \\
\hline BACK & ROUND: & INTER & $=M I N I M A$ & WIN & V-DEFINED & WIN & -DEFINED & \\
\hline & & $n$ & $S_{A}(\%)$ & $n$ & $S_{A}(\%)$ & $n$ & $S_{A}(\%)$ & $\begin{array}{c}E Q 8 \\
\operatorname{CALC~S}_{A^{(\%)}}\end{array}$ \\
\hline$P$ & K & 16 & 24 & 16 & 27 & 16 & 35 & 30 \\
\hline $\mathrm{S}$ & K & 14 & 86 & 16 & 14 & 16 & 15 & 15 \\
\hline $\mathrm{Cl}$ & K & 1 & - & 16 & 25 & 16 & 27 & 22 \\
\hline $\mathrm{K}$ & $K_{\alpha}$ & 14 & 15 & 16 & 1.5 & 16 & 1.7 & 1.1 \\
\hline $\mathrm{Ca}$ & $\mathrm{K}_{\alpha}$ & 16 & 5.2 & 16 & 0.9 & 16 & 1 & 0.7 \\
\hline $\mathrm{Ti}$ & $K_{\alpha}$ & 12 & 43 & 16 & 26 & 16 & 17 & 16 \\
\hline $\mathrm{Cr}$ & $K_{\alpha}^{\alpha}$ & 7 & 140 & 14 & 65 & 16 & 53 & 51 \\
\hline $\mathrm{Mn}$ & $K_{\alpha}^{\alpha}$ & 16 & 3.9 & 16 & 2.1 & 16 & 1.8 & 3.1 \\
\hline $\mathrm{Fe}$ & $K_{a}$ & 16 & 0.9 & 16 & 1.1 & 16 & 1.1 & 1.4 \\
\hline $\mathrm{Ni}$ & $K_{\alpha}^{a}$ & 3 & 54 & 16 & 79 & 16 & 46 & 43 \\
\hline $\mathrm{Cu}$ & $K_{a}$ & 16 & 3.1 & 16 & 6 & 16 & 6 & 4.4 \\
\hline $\mathrm{Zn}$ & $K_{\alpha}$ & 16 & 3.1 & 16 & 3.8 & 16 & 3.6 & 2.8 \\
\hline $\mathrm{Pb}$ & $L_{\beta}$ & 16 & 2.8 & 16 & 3.1 & 16 & 3.6 & 3 \\
\hline As & $K_{\alpha}$ & 16 & 2.6 & 16 & 2.2 & 16 & 1.9 & 2 \\
\hline $\mathrm{Br}$ & $\mathrm{K}_{\alpha}$ & 16 & 4.9 & 16 & 4.9 & 16 & 4.4 & 4.3 \\
\hline $\mathrm{Rb}$ & $\mathrm{K}_{\alpha}$ & 16 & 3.3 & 16 & 4.7 & 16 & 4.6 & 3.7 \\
\hline
\end{tabular}



.

. 


\section{List of Figures}

Figure 1. Comparison of Net Peak Area (N) and Background (B) as Defined in Conventional Background-Subtracted Peak Integration (a) and in Cove11's Direct Peak Integration Method (b).

Figure 2. Comparison of Precisions for a Sma11 Peak Area Determined by Four Integration Methods.

Figure 3. Reduction of Random Error by Repeated Smoothing.

Figure 4. Average Errors in Peak Areas Due to Repeated Smoothing Relative to Areas From Unsmoothed Spectra.

Figure 5. Relative Errors in Peak Areas Before Peak Overlap Correction.

Figure 6. Peak Overlap Errors (Shaded Areas) for (a) Integration with Separately Determined Background, (b) Covell Integration and Background Definition and (c) Covell Integration of a Spectrum Containing Only the Interferring Element Peak.

Figure 7. Variations in Peak Overlap Intensity Near the $\mathrm{Cu} \mathrm{K}_{\beta}$ Peak for Six Analyses of Varying Time Intervals.

Figure 8. Variations in Peak Overlap Intensity Near the $\mathrm{Cu} \mathrm{K}_{B}$ Peak Using Various Window Widths on a Single Spectrum.

Figure 9. Variation in Crustal Abundance-Normalized Alpha Coefficients with the Integration Window Width. 


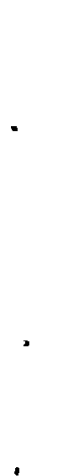


(a)

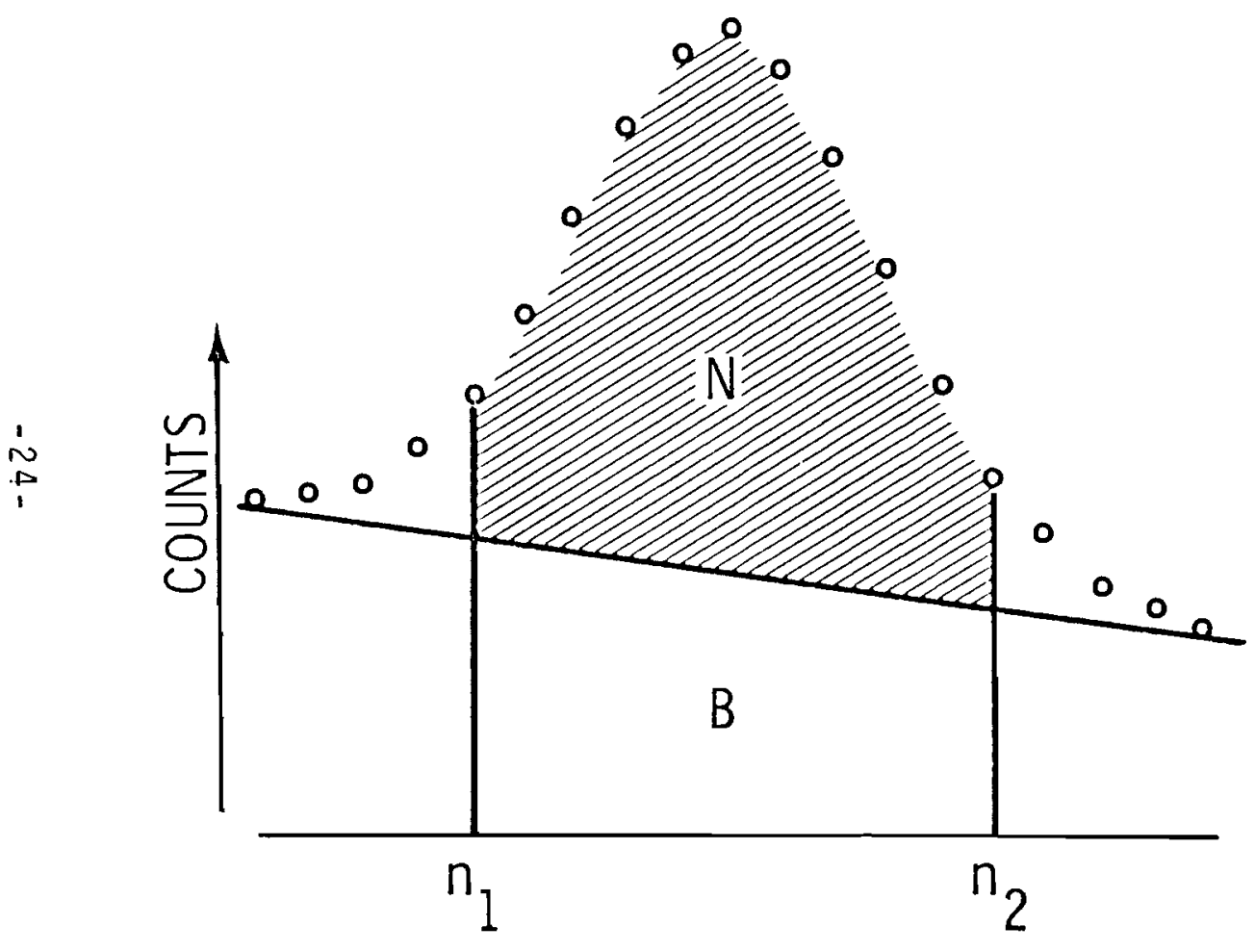

(b)

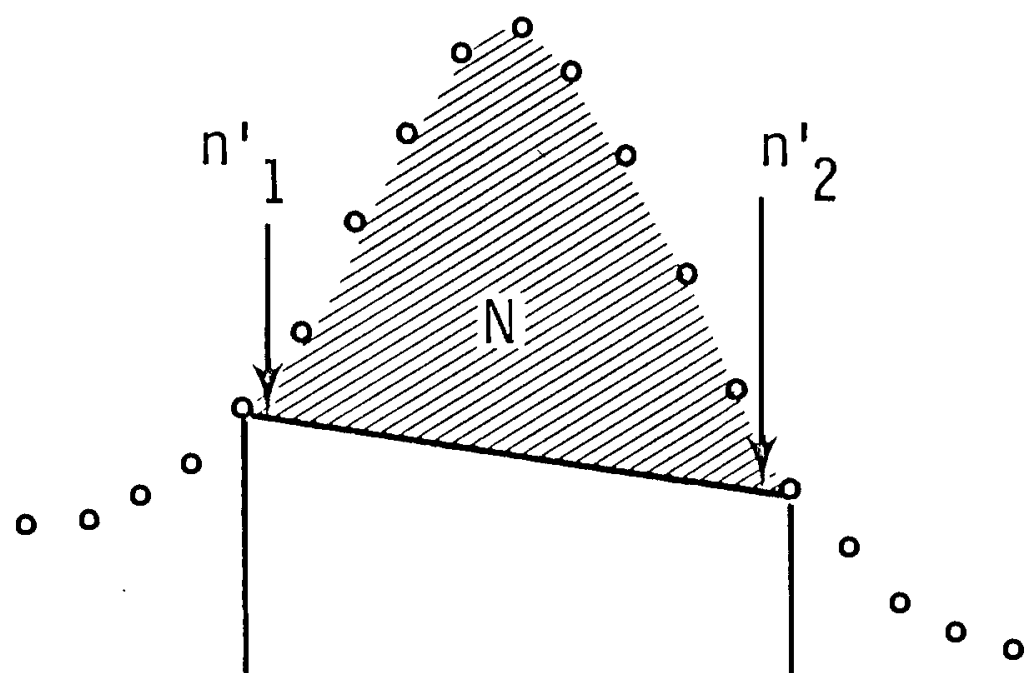

B

CHANNEL NUMBER

Figure 1. Comparison of Net Peak Area ( $N$ ) and Background (B) as Defined in Conventional Background-Subtracted Peak Integration (a) and in Covell's Direct Peak Integration Method (b). 



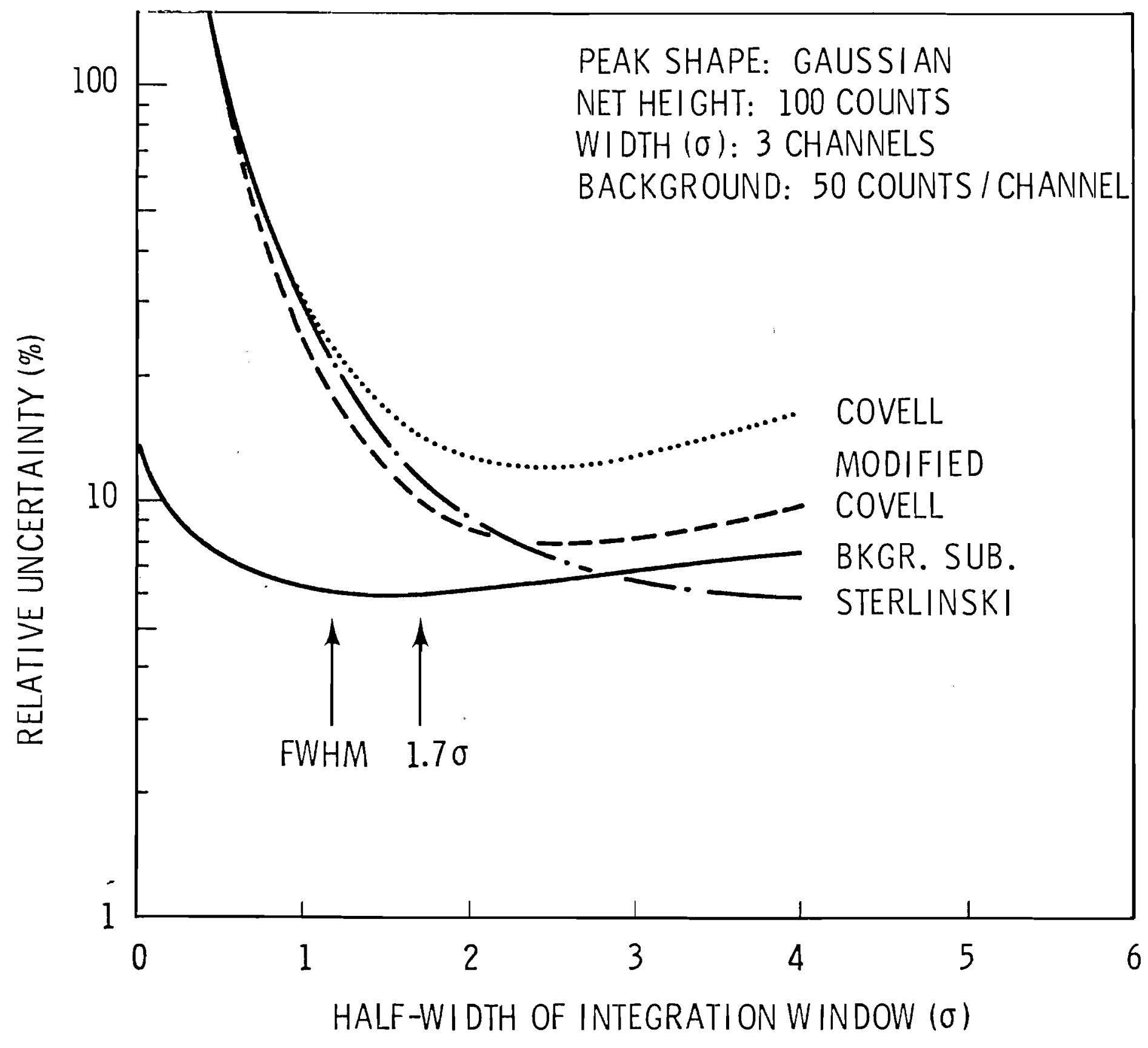

Figure 2. Comparison of Precisions for a Sma11 Peak Area Determined by Four Integration Methods. 


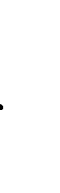




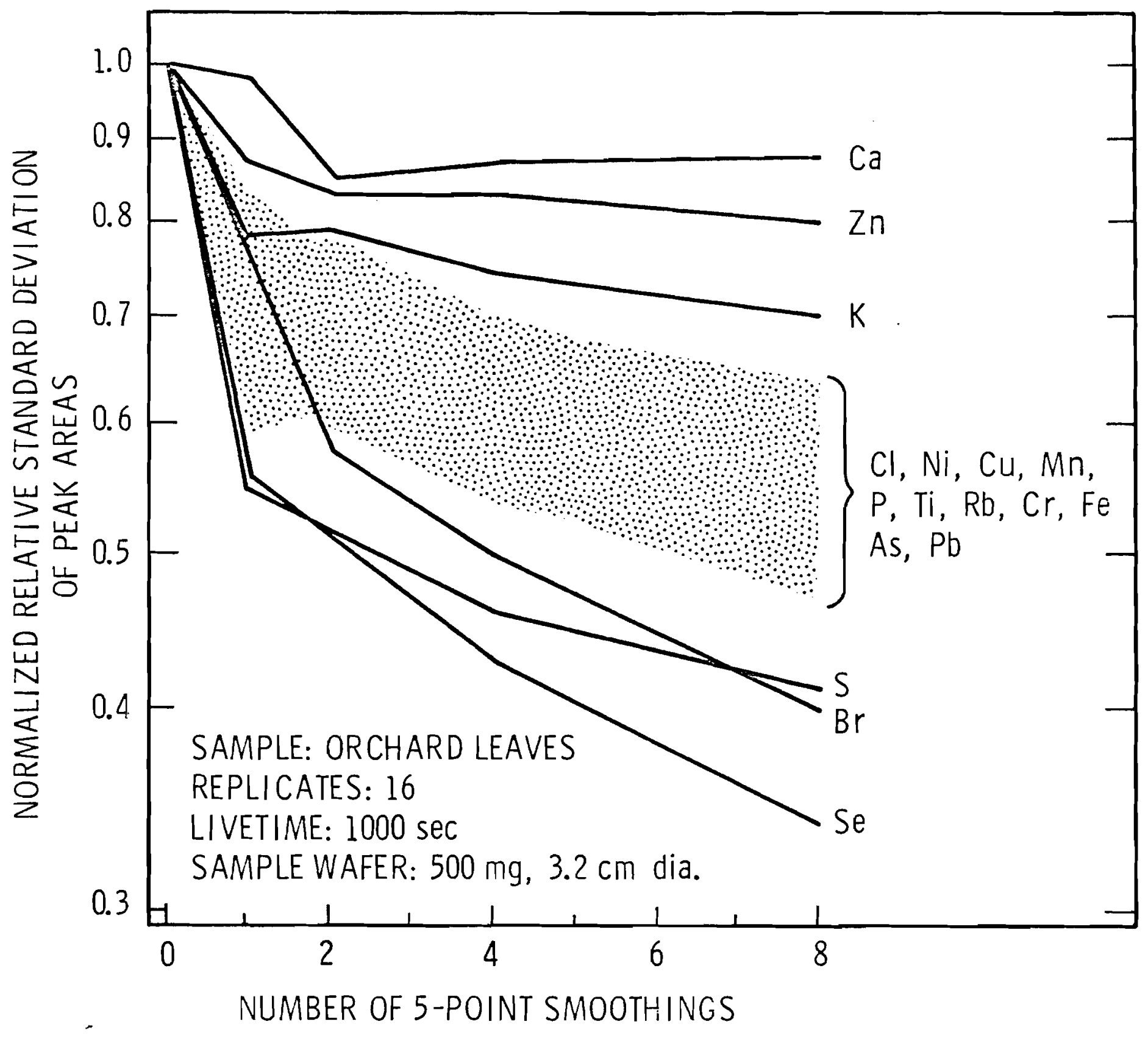

Figure 3. Reduction of Random Error by Repeated Smoothing. 


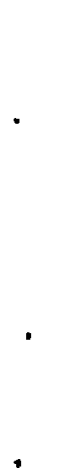




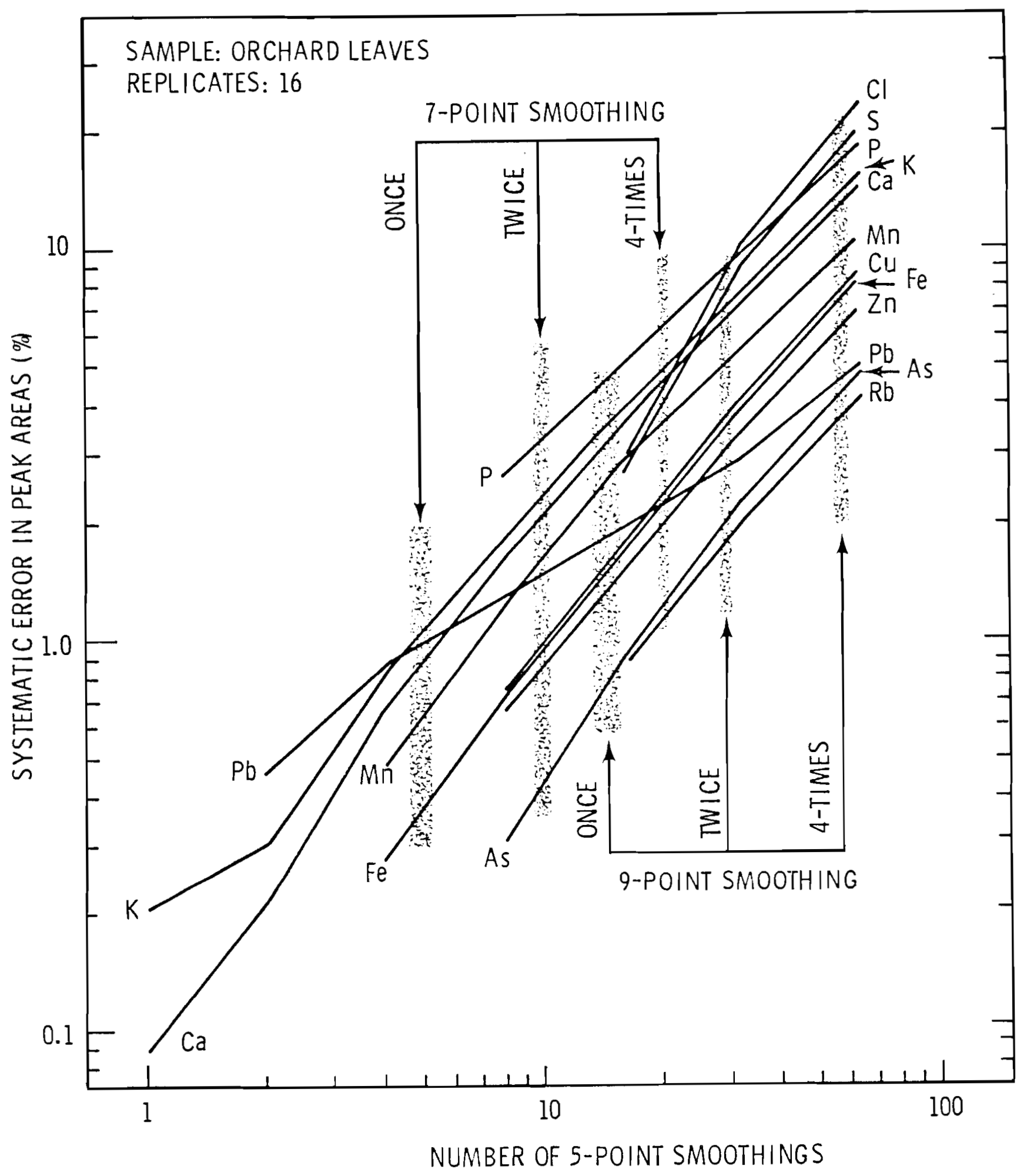

Figure 4. Average Errors in Peak Areas Due to Repeated Smoothing Relative to Areas From Unsmoothed Spectra. 


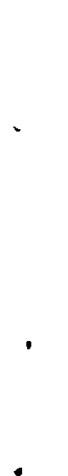




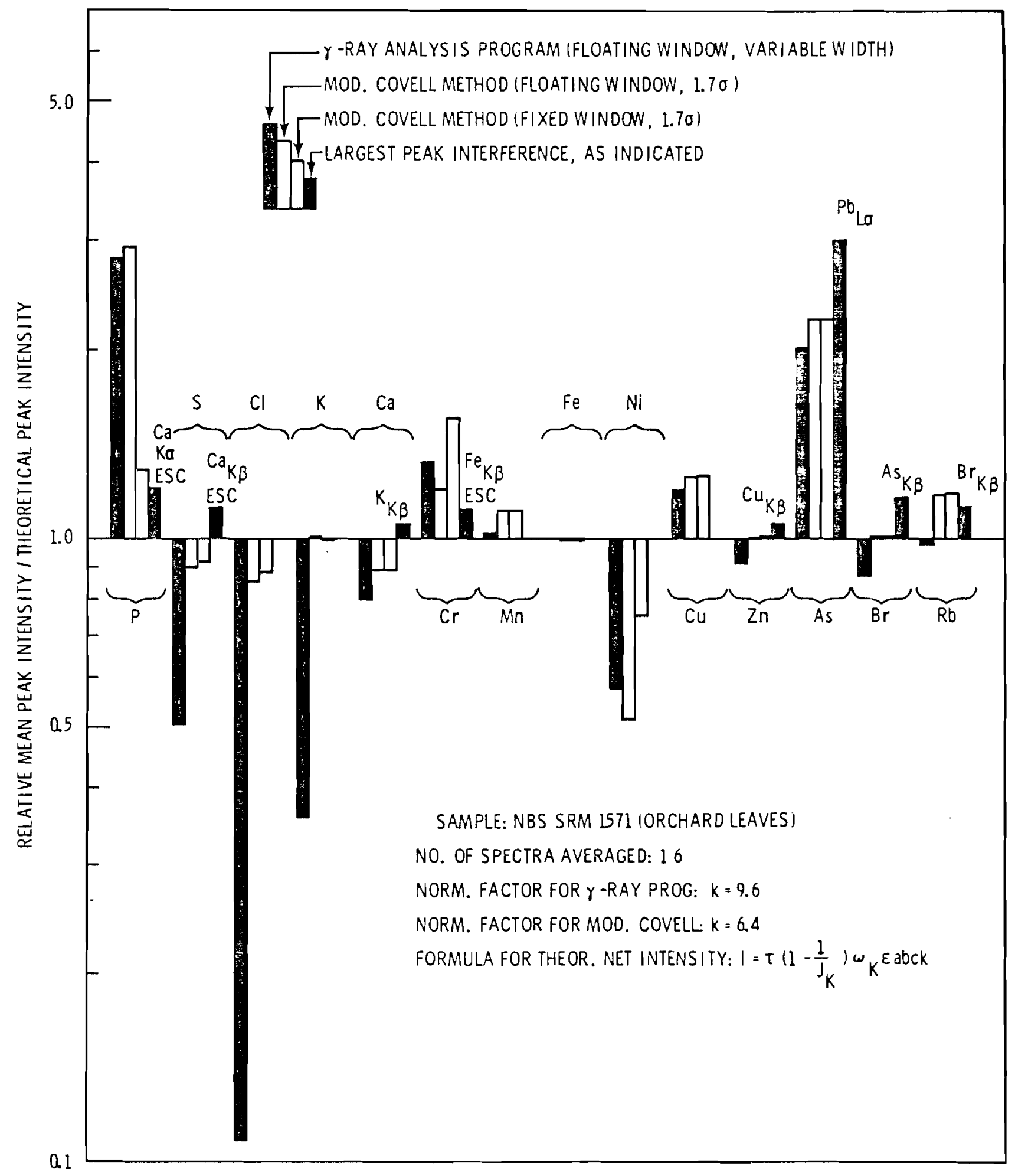

Figure 5. Relative Errors in Peak Areas Before Peak Overlap Correction. 


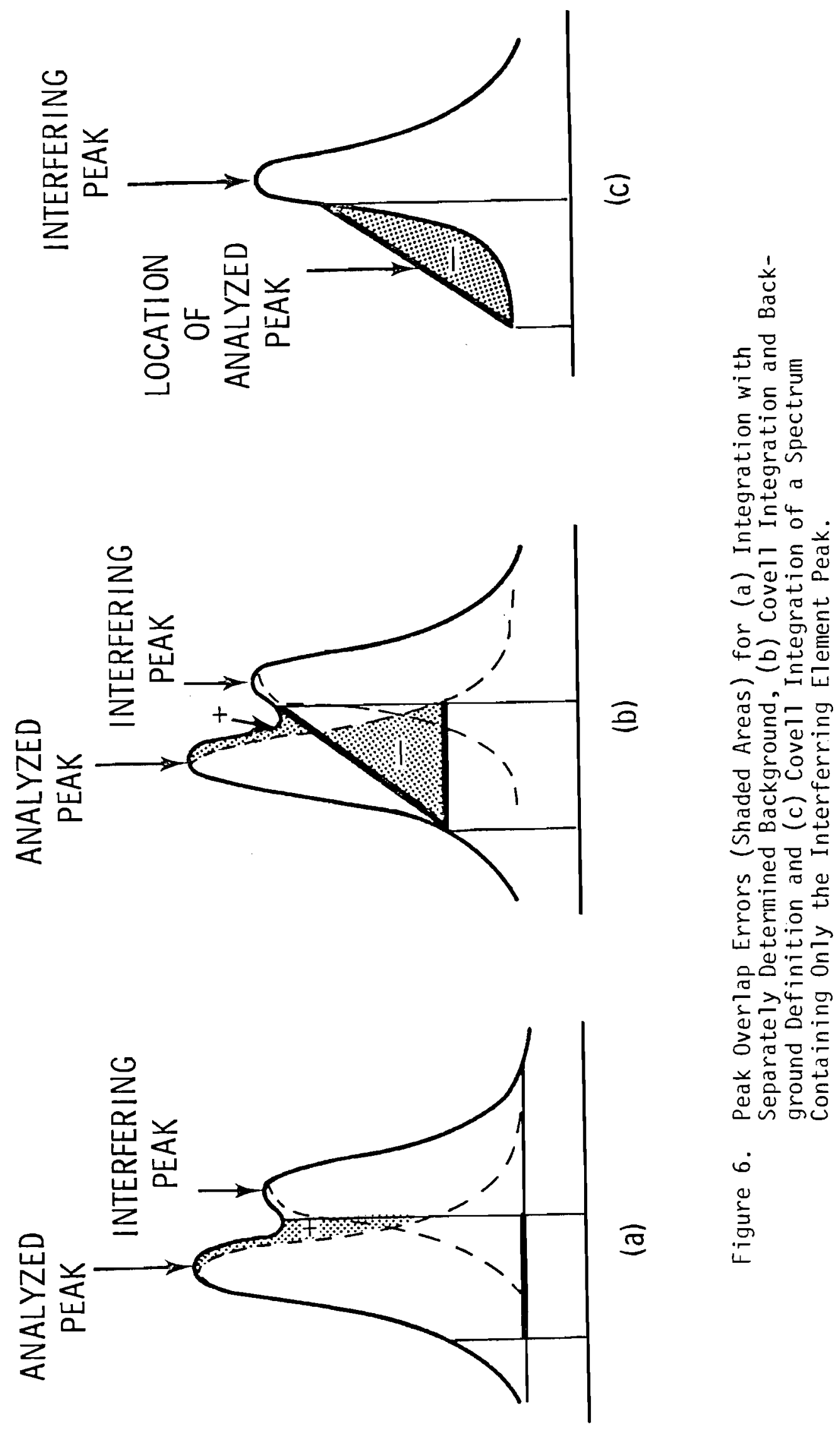


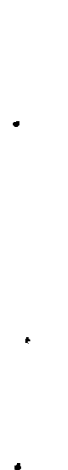




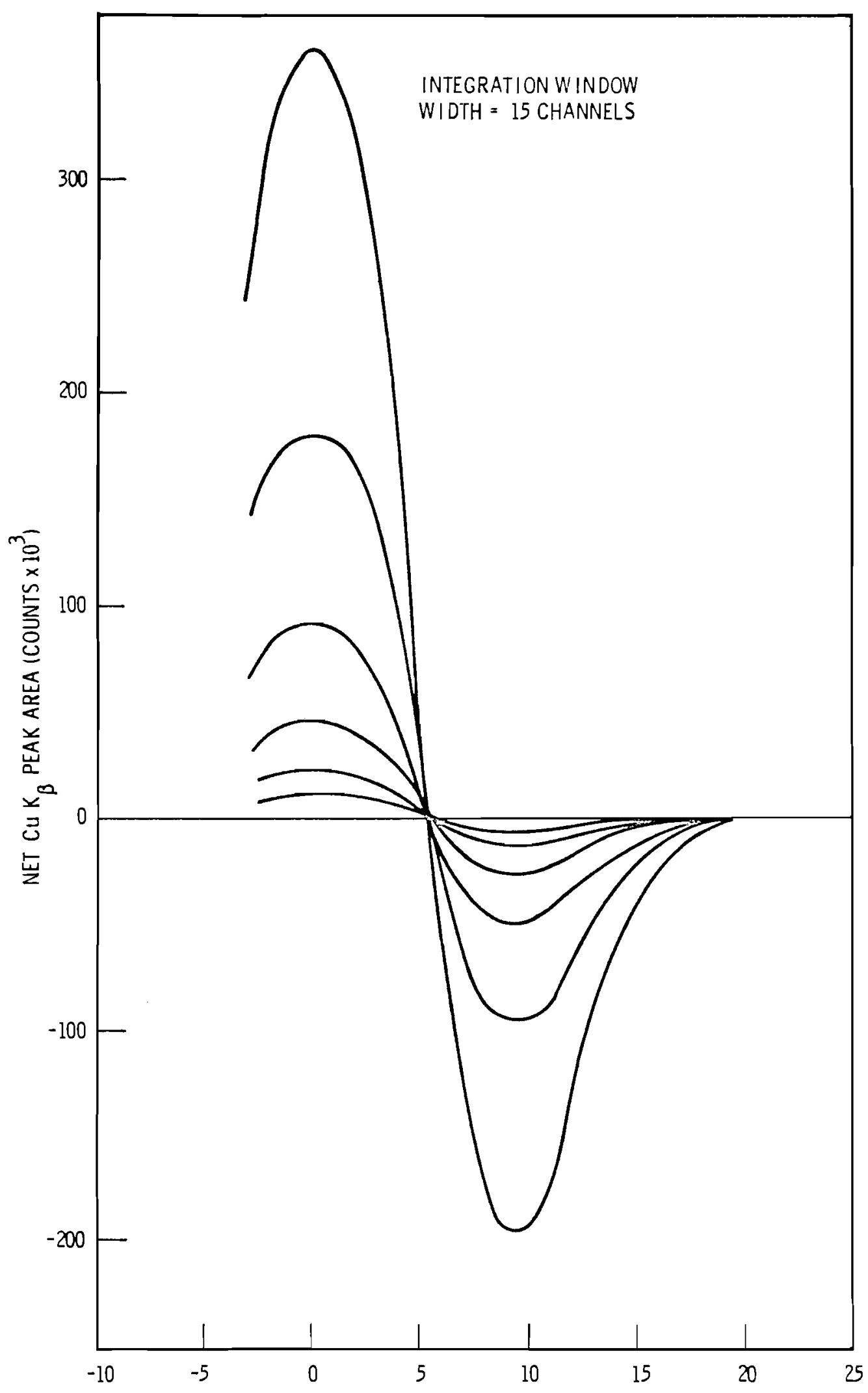

DISPLACEMENT OF INTEGRATION WI INDOW FROM PEAK CENTER (CHANNELS)

Figure 7. Variations in Peak Overlap Intensity Near the $\mathrm{Cu} \mathrm{K}_{B}$ Peak for Six Analyses of Varying Time Intervals.

$$
-30-
$$


. 


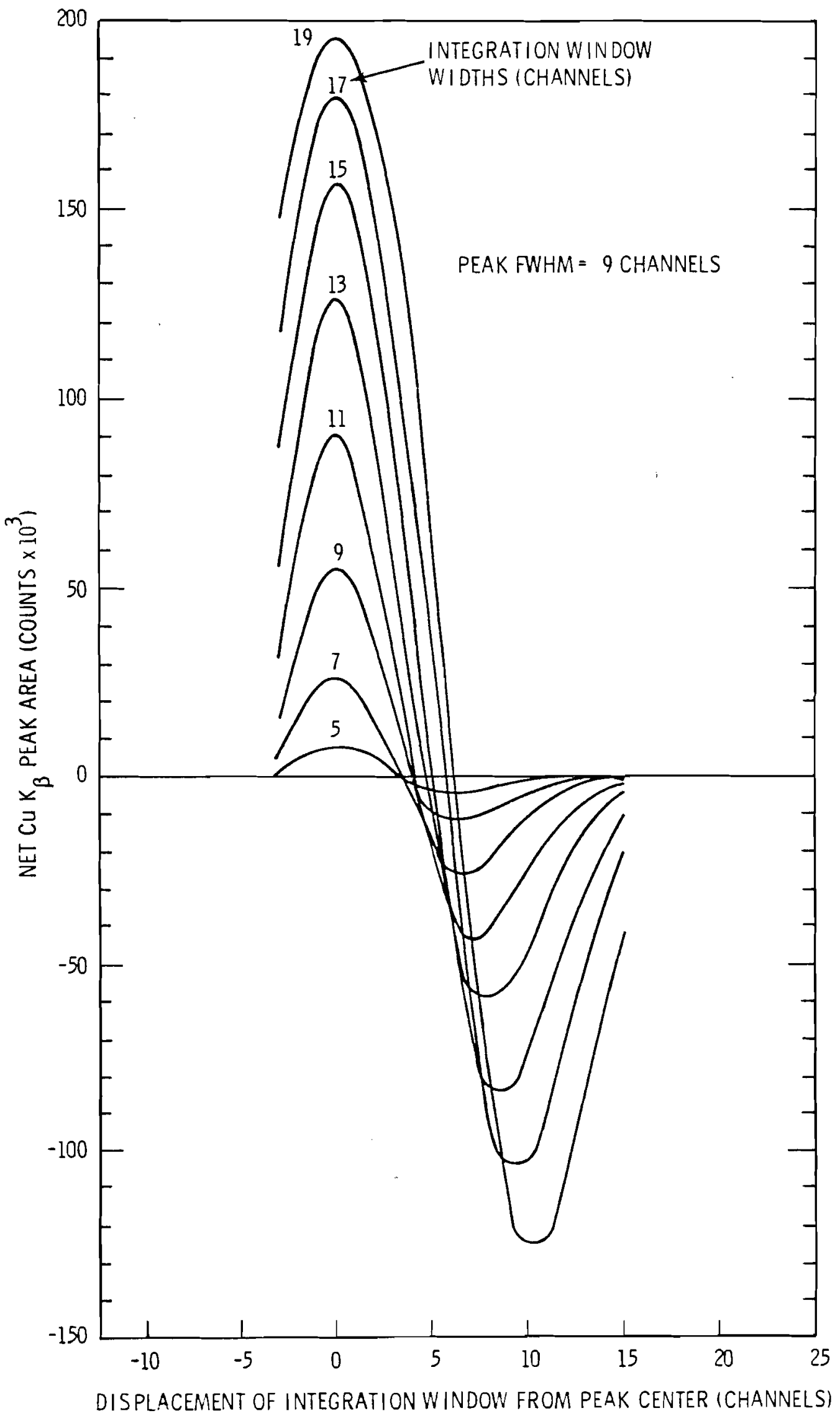

Figure 8. Variations in Peak Overlap Intensity Near the $\mathrm{Cu} \mathrm{K}_{\beta}$ Peak Using Various Window Widths on a Single Spectrum.

$-31-$ 


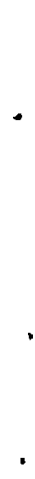




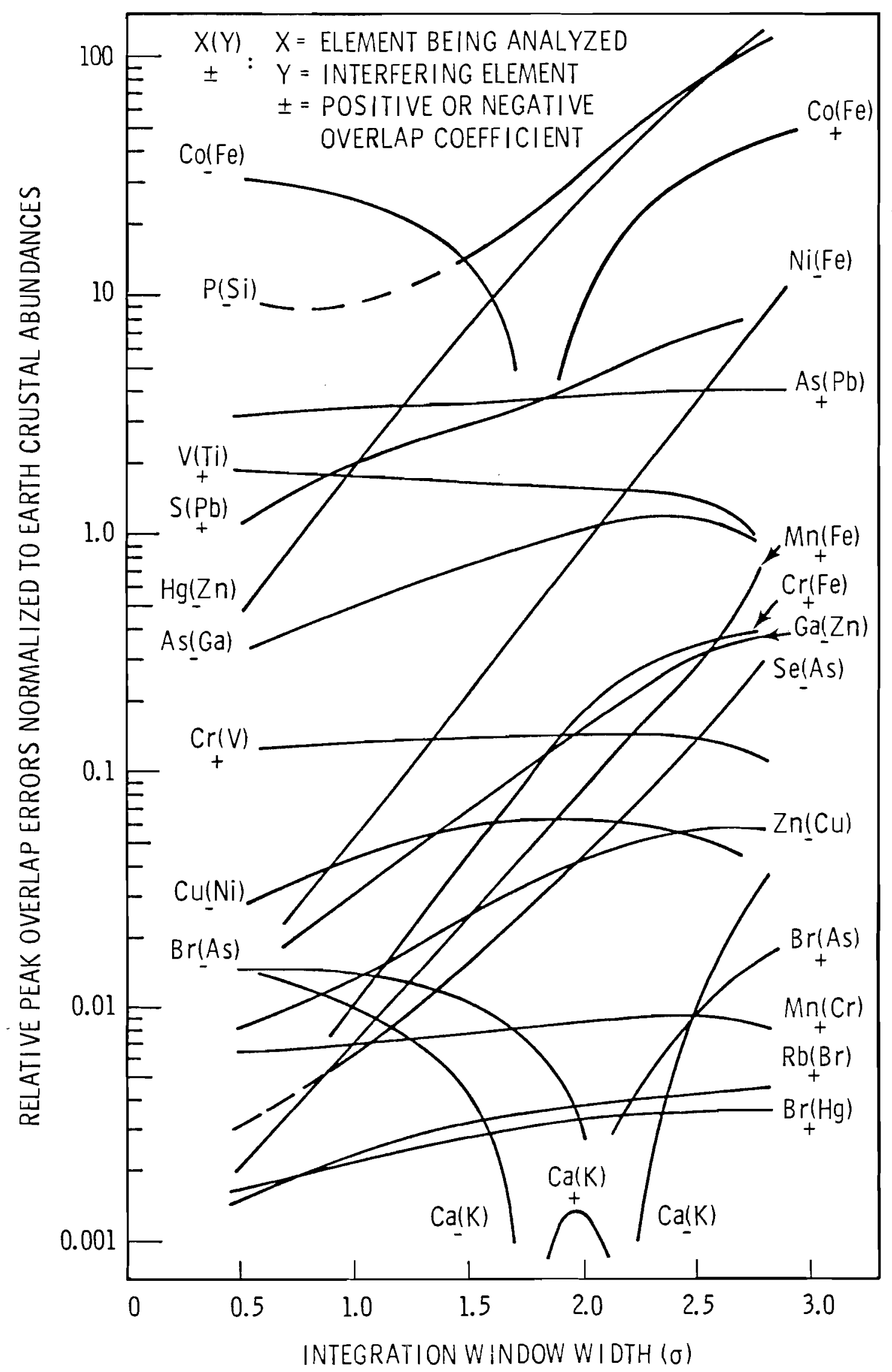

Figure 9. Variation in Crustal Abundance-Normalized Alpha Coefficients with the Integration Window Width. 


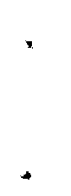


No. of Copies

OFFSITE

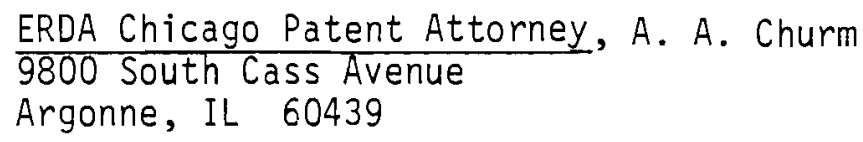

ERDA Chicago Patent Attorney, A. A. Churm 9800 South Cass Avenue

Argonne, IL 60439

ERDA Division of Biomedical and Environmental Research Washington, D. C. 20545

2

ERDA Pittsburgh Energy Research Center 4800 Forbes Avenue

Pittsburgh, PA 15213

1 Ames Laboratory

Iowa State University

Ames, Iowa 50011

Argonne National Laboratory 9700 South Cass Avenue Argonne, IL 60439

2

U. S. Energy Research \& Development Adminstration Brookhaven Area Office Upton, New York 11973

2

University of California Lawrence Berkeley Laboratory Berkeley, CA 94720

$1 \quad$ University of California Lawrence Livermore Laboratory P.0. Box 808

Livermore, CA 94550

2

U. S. Energy Research \& Development Administration Los Alamos Area Office Los Alamos, NM 87544

6

Oak Ridge National Laboratory P.0. Box X Oak Ridge, TN 37830 ERDA Technical Information Center 
. 
ONSITE

8 ERDA Richland Operations Office

H. E. Ransom

21 Battel le-Northwest

J. M. Nielsen

R. W. Perkins

W. D. Felix

R. E. Wildung

G. W. Dawson

K. K. Nielson (10)

Technical Publications

Technical Information Library (5) 
. 